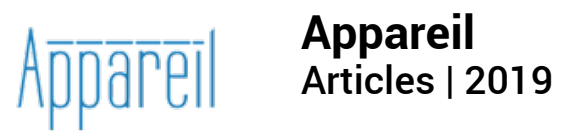

\title{
Comment savoir si c'est de l'art ? Le cas des jeux vidéo
}

\section{Pascal Krajewski}

\section{(2) OpenEdition}

Journals

Édition électronique

URL : http://journals.openedition.org/appareil/3301

DOI : 10.4000/appareil.3301

ISSN : 2101-0714

Éditeur

MSH Paris Nord

\section{Référence électronique}

Pascal Krajewski, «Comment savoir si c'est de l'art ? Le cas des jeux vidéo », Appareil [En ligne], Articles, mis en ligne le, consulté le 30 janvier 2021. URL : http://journals.openedition.org/appareil/ 3301 ; DOI : https://doi.org/10.4000/appareil.3301

Ce document a été généré automatiquement le 30 janvier 2021.

\section{(c) (i) (9)}

Appareil est mis à disposition selon les termes de la Licence Creative Commons Attribution - Pas d'Utilisation Commerciale - Pas de Modification 4.0 International. 


\title{
Comment savoir si c'est de l'art? Le cas des jeux vidéo
}

\author{
Pascal Krajewski
}

1 À quoi bon vouloir montrer que le jeu vidéo est un art? Ou n'en est pas? Pourquoi vouloir le rapporter à une discipline qu'il ne guigne même pas? Se demandera-t-on si le sport est un art? Non, le sport est le sport, et cela va bien à tout le monde. Pourtant, il arrive même au sport de faire usage d'un vocabulaire artistique : tel joueur est un artiste, tel geste, d'une pureté technique inouïe, frise l'art, tel affrontement d'anthologie restera comme un chef-d'œuvre de beau jeu, tel sport joué à ces niveaux d'expertise confine à l'art (escrime, ski), enfin certains sports sont déclarativement artistiques, puisqu'ils visent à la beauté de l'enchaînement et non à l'efficacité du geste (patinage artistique, natation synchronisée, etc.). Le sport pourrait - par hasard, momentanément ou de façon très spécifique - produire des résultats qualifiables d'artistiques, lorsqu'ils allient une parfaite maîtrise technique à une beauté gracieuse. Mais même alors, ils n'en restent pas moins des sports parce que par vocation, ils ne visent pas à être de l'art.

2 A-t-on cherché à montrer que le jeu était un art ? La question est déjà moins fermée et des zones de cousinage ont été identifiées ${ }^{1}$. Pourtant dans la forme, la question recevra le même traitement que celle relative au sport: certains hapax sont de l'art, certains jeux très particuliers, notamment cérébraux ou riches d'une mythologie, peuvent $y$ prétendre (sans doute les jeux les moins ludiques), mais le gros de nos jeux quotidiens sont des jeux, n'ont de vocation que ludique, et ne fraient aucunement avec la sphère de l'art. S'il nous arrive d'évoquer la notion d'«art» dans ce domaine, ce sera par opposition à «technique " ou à "science», pour tenter d'expliquer la réussite outrageuse de certains dans des jeux relevant du hasard par exemple. Si tel joueur de tennis gagne, c'est parce qu'il domine sa technique - si le médecin soigne bien, c'est parce qu'il connait sa science - si le joueur de poker gagne sa vie en jouant, c'est qu'il possède son art. Mais ce n'est là qu'un usage lexical n'ayant pas vocation à installer le jeu dans le champ de l'art. 
Pourquoi la question se pose-t-elle autrement avec le jeu vidéo² ? Sans doute parce que c'est un phénomène d'ampleur et de développement sans précédent; aussi parce qu'il flirte déjà avec des arts voisins (le cinéma, l'animation, la musique), notamment en s'inscrivant dans leur valse de remédiations et d'adaptations ; mais sans doute surtout parce qu'une grande partie des jeux vidéo sont susceptibles de narration, de représentation, voire de poésie. Un contenu culturel, une histoire, un message sont véhiculés par le jeu vidéo. Des émotions, poétiques ou affectives, peuvent naître de son usage. Alors pourquoi des réflexions sur l'intime ou sur le transcendant ne seraientelles pas envisageables? Rien n'interdit a priori au jeu vidéo de produire des "percepts» et des «affects», ce qui définit selon Deleuze la nature de l'art ${ }^{3}$. Rien n'interdirait à un jeu d'être la « manifestation sensible d'une idée » - ce qui définit chez Hegel le Beau, autre nom de l'idéal que l'art a pour mission de déterminer ${ }^{4}$. Rien n'interdirait à un jeu de faciliter « la connaissance de l'Idée, connaissance qui constitue le plaisir esthétique » - ce qui constitue chez Schopenhauer l'œuvre d'art ${ }^{5}$.

Disons-le de façon grandiloquente: le jeu vidéo serait de l'art parce qu'il serait susceptible d'élever l'âme de celui qui y joue. Ou pour le dire de façon moins solennelle : il serait de l'art parce qu'il est capable de poétiser momentanément voire durablement la vie de celui qui y joue, d'être un opérateur de poésie, de rêve, et d'un suspens soudain de la prose du monde.

\section{Prolégomènes à la question}

5 Nous nous demandons si le jeu vidéo est un art, et nous ne savons pas comment gouverner notre questionnement. Comment la question se pose-t-elle? Comment bien poser la question?

6 Demandons-nous, pour débuter, si la peinture est un art? Si je regarde ce qu'a fait l'artisan-peintre dans ma salle de bains, ce que fait mon neveu en classe, ou ce que fait mon papi sur les bords de Seine: je ne vois rien là de plus qu'une activité humaine, professionnelle ou de loisirs, visant à égayer le monde ou la vie de certains individus. Une promenade dans un musée des Beaux-Arts de province, ou pire dans ses réserves, me mettra peut-être sur la voie de l'art sans me convaincre totalement: s'y trouvent entassées des toiles anciennes de peinture à l'huile ou à gouache de paysages, de natures mortes, de scènes bibliques ou mythologiques maintes fois revues. Si cela est de l'art, il est bien morne. Une visite dans une exposition d'envergure internationale, dans un musée de renommée mondiale, me permettra enfin d'entrer en contact avec un certain nombre de toiles et de peintres, triés sur le volet, dont la présence et le souffle s'imposent de pair avec leur évidente maîtrise du médium. Le corps du regardeur s'arrête, transi ; l'esprit est stupéfié ; l'œuvre d'art méduse celui qui ose la braver du regard.

7 De même en va-t-il du cinéma ou de la littérature romanesque. Les résultats des boxoffices instantanés indiquent les best-sellers ou les blockbusters que la critique savante a tendance à dénigrer ; le gros de la production concerne des travaux sincères et moyens, qui auront peu d'audience parce qu'ils ont peu d'intérêt ou parce que leur auteur n'est encore qu'en devenir. Enfin, quelques fois l'an, une œuvre sort du lot, s'impose par son caractère universel, profond et riche, sa mise en forme impeccable et un sujet à la hauteur. Alors on saluera le chef-d'œuvre; alors on s'inclinera devant l'œuvre d'art. Semblablement, si l'architecture est un art, ce n'est pas en vertu des innombrables 
édifices du commun qui viennent scarifier nos paysages et nos villes; ce n'est que relativement aux rares monuments où un geste technique s'allie au souci de faire lieu et de durer.

Dire que la peinture, le cinéma, la littérature ou l'architecture sont de l'art, c'est donc ne sauver qu'une infime partie de leur production, et passer sous silence tout le reste comme étant de la "sous-littérature", des "produits de consommation», du " divertissement », de "l'art de masse ». Est donc considérée comme un art, une discipline technique productrice de contenus intelligibles et sensibles, dont certains travaux parviennent au statut d'œuvres d'art, en démontrant des qualités littéralement exceptionnelles. Et celui qui voudrait insister sur le peintre du dimanche, le film à gros budget ou le livre à l'eau de rose pour prouver que cinéma, peinture et littérature ne sont pas des arts, se verrait renvoyer dans ses cordes, son argumentation jugée indigente, sophistique et inacceptable.

9 À l'inverse, certains arts n'arrivent pas à s'élever au-dessus du statut d'«arts appliqués ». Cette classification est d'ailleurs évolutive et partiellement socioculturelle. Le groupe des arts installés dans leur panthéon, n'a pas toujours été celui que l'on connaît et n'est pas universel. En Orient, la cérémonie du thé, l'art des jardins ou l'ikebana voisinent avec la calligraphie et la peinture sans que personne ne s'en offusque. Nos arts plastiques actuels - peinture, sculpture, architecture - ont été déconsidérés jusqu’à la Renaissance (parce que « mécaniques »). Au contraire, parmi les neuf muses, se tient celle de la pantomime, délaissée de nos jours. Pourquoi l'art du vitrail, de la tapisserie ou de la mosaïque, qui produisent des chefs-d'œuvre depuis des siècles, dûment embaumés dans nos musées et nos lieux de mémoire, n'ont-ils pas intégré le groupe très sélectif des beaux-arts ? Pourquoi la céramique ou l'orfèvrerie n'en sont-ils pas, eux dont nos musées d'antiques regorgent? Serait-ce parce que l'art aurait obligation de représentation? Mais le vitrail s'y emploie et l'architecture pas du tout. Serait-ce parce que l'art ne saurait se compromettre dans des productions utiles? Dans ce cas la céramique et le design ne pourraient y rêver, mais pas moins que l'architecture.

10 Les beaux-arts sont nés avec Vasari, au XVI ${ }^{\mathrm{e}}$ siècle $^{6}$. Certains arts mécaniques cherchent alors à se distinguer du vulgaire artisanat et se haussent du col pour accéder au statut des « arts libéraux ». L'auteur italien argumente en plaidant que peinture, sculpture et architecture peuvent prétendre à une forme d'idéalité artistique, dans la mesure où toutes trois se fondent sur le dessin, et plus précisément sur le disegno. Il suffit en effet de donner toute son extension au terme italien pour parvenir à réunir le dessin et l'idée : le disegno est l'expression visible d'une idée, la manifestation d'un concept que l'on a à l'esprit. Or l'art vise l'Idée, donc le disegno, mi-matériel mi-spirituel, oscillant entre dessin et dessein ${ }^{7}$, relève de l'art - et les disciplines qui en découlent rentrent en odeur de sainteté. L'Accademia del Disegno de Florence est fondée en 1563 (ce sera la première du genre en Europe) : on y enseigne la peinture, la sculpture et l'architecture.

11 En regard, les arts appliqués se définiront comme ceux lestés par leur attachement à une matérialité trop pesante ou trop imprécise. Le céramiste est un artiste grossier du corps et du geste, le mosaïste ne peut composer que des images granulaires de résolution limitée. Parce que le poids de la matière ou de la technique les empêche de gagner une totale liberté de représentation, ces arts se cantonnent à la rubrique de l'appliqué. Pas sûr qu'à pareille époque, le cinéma, toute fragmentée qu'est sa production, eut atteint au rang d'art... Quant à la poésie, à la musique, à la danse et au 
théâtre, ils sont considérés depuis l'Antiquité grecque comme des arts parce que justement non pollués par une quelconque facture artificielle.

La distinction entre beaux-arts et arts appliqués reste forte dans nos sociétés, notamment à cause de son maintien dans nos écoles et nos musées. Les étudiants des arts appliqués trouveront "du travail», dans des branches de l'industrie; ceux des beaux-arts seront condamnés à devenir des "artistes ", courant les galeries pour faire reconnaître leur génie. Toute discipline tirant vertu du fait de fournir des débouchés industriels à ses étudiants, toutes créatives et modernes que soient ces industries, ne pourra s'élever au-dessus du rang des "appliqués ». Pourtant n'est-ce pas exactement la situation du cinéma, auquel des écoles très sélectives fournissent moult techniciens ? N'est-ce pas encore assez semblable à ce qui se passe dans nos conservatoires de musique, qui, pour une minorité de compositeurs et de chefs d'orchestre, forment des cohortes de musiciens virtuoses qui ne sont d'une certaine manière que les moyens anonymes de manifester l'œuvre singulière d'un autre?

Pour tenter de saisir au mieux la différence entre "beaux " arts et arts " appliqués ", on peut en appeler à l'un des sous-domaines de ce dernier : les arts décoratifs. Car les arts appliqués sont décoratifs d'abord, ils cherchent à égayer, à ornementer (dirait Loos $\left.^{8}\right)$, des produits du quotidien. Dans cette perspective les vitraux ne sont que des vitres enjolivées, les mosaïques des sols décorés, la céramique de la vaisselle embellie. Un intérêt poli est attendu vis-à-vis de leurs produits - non une contemplation réfléchissante. Porter un bijou, boire dans une tasse, valser dans une robe, marcher sur un parterre seraient les régimes normaux de réception de ces produits d'art appliqué, un régime d'usage, où la contemplation et le plaisir du Beau n'ont pas lieu d'être et ne sauraient se manifester que sous une forme très dégénérée. Sera donc considéré comme un art, un champ du sensible dont la réception spécifique invite à une attention et une attitude esthétiques, appréhendant l'œuvre comme un miroir de l'homme, du monde ou de l'Absolu.

Mais la donne semble avoir été totalement bouleversée au $\mathrm{Xx}^{\mathrm{e}}$ siècle, de sorte que ces vieux paradigmes (de l'art puis de l'artisanat) n'auraient même plus cours, rendant impossible tout recours à une quelconque nature de l'art ou à quelques critères identificatoires. En effet, le xx ${ }^{e}$ siècle aura connu deux révolutions qui ont fait éclater la sphère de l'art.

La première est interne, c'est l'aventure des arts moderne et contemporain, portée par un discours critique esthétique et philosophique de plus en plus dominant. Elle débouche sur un monde de l'art où "tout vaut", tout peut faire art, tout fait art. Impossible dès lors de refuser ce statut à telle ou telle œuvre en vertu de sa forme, de sa qualité ou de quoi que ce soit. La théorie institutionnelle de l'art, malgré la modestie de ses propositions, s'impose alors pour penser l'art, reléguant aux oubliettes les discours plus ambitieux sur le sens, la transcendance, la beauté et consorts.

16 La seconde est externe, c'est l'explosion de la culture mass médiatique, avec ses nouvelles technologies de production de contenus: photographie, cinéma, enregistrement sonore, vidéo, ordinateur, internet, etc. À présent, «tout se vaut », la culture, voire la simple production de contenus médiatiques a rang d'art ou plutôt démonétise la question de l'art, en la reléguant comme non pertinente. Car tout ce qui est produit par une technologie médiatique serait d'un impact plus important sur nos vies et notre sensibilité que l'art élitiste des musées ou des théâtres - et c'est cette offre-là qu'il convient à présent d'analyser pour tirer les grilles de valeurs auxquelles l'art immémorial devra répondre. La sphère de l'art implose devant la pression de la 
culture de masse qui impose ses outils, ses rythmes, ses vertus, ses critères - et son économie.

Et pourtant... Et pourtant, ces nouveaux paradigmes dominants de l'articisation généralisée ${ }^{9}$ et des médias ${ }^{10}$, secondés par une approche théorique pragmatique et contextuelle (grandement anglo-saxonne), ne closent pas le débat. La question de l'art n'est pas résolue, elle est abandonnée. Comme s'il était soudain plus honorable d'arrêter de fixer les vieilles lunes pour s'enticher des doigts boudinés qui les dardent. Car l'art ni les hiérarchies n'ont pour autant disparu. Certaines productions sont meilleures que d'autres; certains œuvres dureront, quand l'écrasante majorité de ce qui est produit périra sans perte; certains travaux du monde de la culture continuent de nous émouvoir, nous ouvrir les yeux, modifier notre sensibilité, nous épanouir ou nous inquiéter. L'art y est donc bien encore présent sous sa forme archaïque, immémoriale, qu'il faut continuer de traquer, de penser, armés de nouveaux outils comme des anciens (puisque malgré tout, l'essence de l'art se survit au cours des siècles et des changements d'époques).

Le jeu vidéo intervient donc, à ce stade de l'histoire de l'art et dans ce contexte technologique, comme le point nodal où doivent se confronter pour s'articuler ces différentes approches esthétiques. En cela il fait époque, et pour des raisons plus que techniques: industrie spectaculaire nécessitant de nombreux savoirs appliqués, forgeant un imaginaire de culture geek de plus en plus partagé, s'installant au centre du jeu des productions médiatiques, et parfois mis en avant pour accéder au rang d'art. Le jeu vidéo émerge comme cette terra incognita où loisirs, médias, imaginaire, culture et art viennent frayer anarchiquement. Une zone difficilement cartographiable car il y faut les outils (parfois contradictoires) de tous ces analystes pour la décrire, mais qui prend de plus en plus d'ampleur.

19 Le présent article n'est qu'introductif. Après avoir rappelé les enjeux, il vise à dresser un état des lieux, modeste, de la question de la présence de l'art dans le jeu vidéo, ou encore de sa nature artistique. Il remet au centre la question laissée de côté par l'approche médiatique, non certes pour la résoudre, mais pour la présenter comme la question première du point de vue philosophique, et essayer de trouver des biais pour la prendre, en conciliant les outils et les usages nouveaux avec les enjeux et la nature profonde de l'art. Observons donc les différents moyens utilisés par le jeu vidéo pour, quatre siècles après la peinture, la sculpture et l'architecture, briguer cette dignité...

\section{Enrôlement dans un contexte artistique institutionnel}

Le prestigieux Museum of Modern Art de New York a fait entrer une dizaine de jeux vidéo dans son département Architecture et design en 2012. En 2013, deux jeux vidéo sont accueillis dans le département «Film et médias» du Smithsonian Art Institute (Washington). A priori, la question semble donc tranchée pour ces deux institutions artistiques de premier plan. Notons cependant leur différence de point de vue.

21 Pour la première, les jeux vidéo relèveraient du design, et seraient du design appliqué, ce que les Anglo-Saxons appellent encore du design interactif ${ }^{11}$. La conservatrice du musée qui a réalisé cette audacieuse introduction insiste en effet sur les qualités ergonomiques, voire informatiques de ces programmes vidéoludiques :

Nos critères mettent en avant non seulement la qualité visuelle et l'expérience

esthétique de chaque jeu, mais aussi les nombreux autres aspects - de l'élégance du 
code au design du comportement du joueur - qui se rapportent au design d'interaction ${ }^{12}$. de l'art en est pleine, démontrant leur inutilité : les polémiques passent, leurs objets s'enracinent. Regardons cependant de plus près le MOMA. Le musée est globalement coupé en deux, aux deux derniers étages dominent la Peinture et la Sculpture, aux deux premiers étages sont rangés le reste des collections, à savoir les départements Architecture et design, Arts graphiques, Photographies, Imprimés et Médias. Si l'on jette un coup d'œil rapide sur la liste des œuvres du département Architecture et design, on s'aperçoit qu'il joue le rôle de musée d'arts appliqués voire d'arts et métiers. L'architecture y voisine en effet avec les véhicules, les logiciels, les textiles, le mobilier, les appareils ou les jouets. L'estampille MOMA est donc loin de suffire pour qualifier d'artistique tout ce que son musée recèle... Et défendre que le jeu vidéo a sa place au MOMA $^{14}$, n'est pas dire qu'il relève de l'art ${ }^{15}$... Quant à la démarche du Smithsonian, plus en phase avec une vision esthétique dans laquelle l'art contemporain conduit à l'art des nouveaux médias, donc à l'art électronique et à certains usages des jeux vidéo, elle n'élit bien qu'un type très particulier de jeux, et qui sont peut-être dans une certaine mesure à peine des jeux vidéo ${ }^{16} .$.

En Australie, en 2012, c'est l'Australian Center of Moving Image, une sorte de cinémathèque élargie (dont l'objet est le cinéma, la télévision, le jeu vidéo et la culture numérique), qui monte l'exposition «Game masters ». Les deux approches précitées se trouvent alors mêlées puisque les trois sections mettent respectivement en avant les 
jeux qui marquèrent l'histoire, les créateurs qui défièrent leur temps par des jeux particulièrement innovants et la scène indépendante (avec des titres devenus pour certains des standards : Minecraft, Angrybirds).

Entrant dans des institutions culturelles légitimes ${ }^{17}$, le jeu vidéo, objet hybride et mal classable, peine quand même à se frayer un chemin jusqu'à ses départements classiquement artistiques $^{18}$.

\section{Analogie avec des arts connexes reconnus}

Un autre biais pourra consister à rapprocher l'impétrant d'arts reconnus. Si le candidat partage avec tel art suffisamment d'éléments fondamentaux, il doit pouvoir acquérir le statut d'art au même titre que celui-ci - par capillarité. Si l'on considère le pan " narratif » des jeux vidéo ${ }^{19}$, on peut très aisément les définir en insistant sur le fait qu'ils sont un «film ou un roman dont vous êtes le héros » (ou dans le cas des jeux en réseau, un "protagoniste »). Si l'on considère le pan "mécaniste » ou «systémique » des jeux vidéo ${ }^{20}$ (les sports, les jeux de puzzle, voire les jeux sans but à la Minecraft ou Simcity), on peut le définir comme la manifestation sensible d'un schème qui confine soit à une " performance artistique dont vous êtes le danseur-chorégraphe » soit à une " édification architecturale dont vous êtes le maître d'œuvre " ${ }^{21}$.

Par conséquent, si un film peut émouvoir, un jeu vidéo le pourra aussi - si un livre peut faire réfléchir, le jeu vidéo itou - si un monument peut vous ébranler ou si une toile ou une danse peuvent vous faire vivre un moment d'Absolu, le jeu vidéo doit pouvoir y arriver.

La remédiation des œuvres entre ces différents média peut avoir ici valeur de preuve massue voire de véritable pierre de touche : si l'œuvre Salammbô (ou Alice aux pays des merveilles ${ }^{22}$ ) est déclinable sous format $\mathrm{BD}$, cinématographique, animé, ou vidéoludique - alors ces média présentent tous les qualités suffisantes pour, chacun dans leur régime, offrir une expérience délectable de l'œuvre Salammbô. Celle-ci pourra trouver, dans chacun de ces moyens d'expression, des ressources spécifiques pour se déployer de façon variée tout en conservant son principe génétique et son identité nominative. Le problème, c'est que le merchandising démontre que l'univers d'une œuvre peut se prolonger bien au-delà du champ artistique : les jouets Star Wars ne seraient pas de l'art même si la saga de Lucas l'était. Certes, un jouet n'est pas véritablement une déclinaison d'un film, mais que penser d'un jeu de plateau qui reprendrait le scénario d'un film (type Indiana Jones ou Sherlock Holmes) ? Il en serait bien une remédiation, mais dans un champ assurément non artistique.

Par ailleurs, le recours à des techniques de production similaires plaiderait dans le même sens. Ainsi de l'utilisation de la motion capture qui permit au jeu vidéo d'intégrer dans la trame de son récit des séquences filmées avec des acteurs (cinématiques) - et qui s'est largement répandue dans le champ cinématographique (Avatar). Parce qu'ils font appel à des vedettes du cinéma (en tant que telles) et parce qu'ils mettent en boîte des plans et des séquences de la même façon, cinéma et jeu vidéo partagent un ADN commun $^{23}$. De même pour l'écriture scénaristique, que l'on retrouve dans le film, le jeu vidéo, la bande dessinée ou le roman. S'abreuvant aux mêmes sources, ces disciplines sont cousines et la fonction artistique des unes doit échoir en partage à la dernière venue. 
31 Version amoindrie et dégénérée de cet argument : si l'impétrant utilise suffisamment d'éléments artistiques d'autres arts, il doit pouvoir acquérir le statut d'art. En effet, si on le décortique, le jeu vidéo amalgame de la fiction (comme le roman), de la représentation (comme la peinture), de la musique, du montage et de la cinématique (comme le cinéma). C'est donc une chimère d'arts parfaitement installés, et on supposera que la somme d'arts doit en être un. L'opéra n'est-il pas la somme de trois arts, théâtre, chant et musique?

L'argument de la «capillarité artistique » ne pourra être manié qu'avec parcimonie, puisque dans le même temps, il faudra montrer que le jeu vidéo est un art sui generis, élaborant son propre médium, c'est-à-dire possédant ses propres ressorts, enjeux, moyens d'accéder à la fonction artistique. C'est alors aux notions d'interactivité ${ }^{24}$, de gameplay ou de fun ${ }^{25}$, que l'on renverra pour mesurer les qualités esthétiques du jeu - ou à celles de système de jeu et de game design pour expliciter la conception et la vision du créateur $^{26}$. Mais en quoi de telles spécificités invalideraient-elles la prétention artistique de la chimère vidéoludique?

33 Avantage tactique d'une telle démarche: profiter de l'histoire mouvementée mais décisive de ces disciplines reconnues tardivement comme arts. Que ce soit la photographie, le cinéma, la bande dessinée ou le jazz - les archives sont pleines des cris horrifiés poussés par les plus hautes autorités de leur temps, les dévaluant et les vouant aux gémonies. Et pourtant, qui aujourd'hui refuserait leur intronisation dans le panthéon artistique? Dès lors, la méthode la plus sûre serait de faire le gros dos: laisser les conservateurs hurler; dans une ou deux générations, leurs enfants auront été élevés aux biberons de cette nouvelle invention et ils se feront fort de la légitimer doctement. Nous y sommes.

\section{Intégration dans un système des beaux-arts élargi}

34 On pourrait encore choisir de défendre le caractère artistique du jeu vidéo en lorgnant du côté des activités subalternes, primitives ou exotiques qui furent adoubées par l'art (notamment au xx siècle) : c'est le cas de l'art des fous, des arts premiers, de l'art des jardins, etc. L'Art a su leur ménager une place dans sa trame même (certes dans ses franges), en les rattachant à des grandes familles: la peinture, la sculpture ou l'architecture - en vertu de principes archaïques qu'ils exemplifieraient eux aussi.

On pourrait s'étonner de trouver dans le Système des beaux-arts d'Alain, paru en 1926, le costume, la mode, l'art équestre, le cirque et même la politesse prendre rang sous la discipline "danse et parure»; tout comme le mobilier, le design, le jardin, et l'urbanisme émargent au chapitre de l'architecture. Quant à Kant, il avait introduit l'art des jardins comme pendant de la peinture en tant qu'art qui «présente l'apparence sensible artistiquement liée avec des idées ${ }^{27}$ ». L'art de peindre se trouve d'ailleurs élargi, chez le Maître de Konigsberg, à tout ce qui a trait à la décoration et à l'ameublement, jusqu'à l'art de s'habiller! Retenons cependant que ces arts cousins, de second rang, sont rattachés à l'art princeps en vertu de principes transcendantaux. C'est parce que peuvent s'y exprimer certaines idées sensibles, ou parce qu'ils visent au libre jeu de l'imagination avec les Idées et à « occuper sans fin déterminée la faculté de juger esthétique »-que ces arts sont reconnus comme tels. Il serait alors loisible de rattacher 
les jeux vidéo à des principes artistiques avérés ayant déjà vu bourgeonner de nombreux fruits plus ou moins fidèles. Par exemple, en suivant Alain ${ }^{28}$.

En tant qu'il est un appareil technologique, avec lequel le récepteur est en interaction, il pourrait être intégré à la notion de design, et à plus d'un titre. En effet, il recourt au design matériel (celui des périphériques) mesurant la qualité des joysticks et capteurs en tout genre et leur facilité d'usage - au design numérique (celui de l'interface graphique) appréciant l'habillage des informations extra-diégétiques (menus, barres d'états, fenêtres contextuelles) - au design informatique, saluant la qualité du code du programme, sa bonne facture, la robustesse ou l'ouverture de son «moteur de jeu »enfin, au game design, jugeant l'ensemble des éléments visibles du jeu concourant à sa jouabilité (architecture des bâtiments, accessibilité des énigmes, circuits et trajectoires imposées, mouvements autorisés, degré de liberté offert, etc.). Derrière le mot design, il faut donc lire ici la capacité de schématiser des déroulements ou des interactions avec le monde simulé et ses outputs - ce qui le rattache bien à l'art de l'architecture.

En tant qu'il est un divertissement audio-visuel, il serait rattaché à la grande famille des médias, aux côtés de la télévision, de la radio, de la vidéo, de la Hi-Fi, etc. ${ }^{29}$. On pourrait alors s'intéresser à la richesse de l'intrigue et aux moyens proposés pour la suivre (signalétique, actants auxiliaires), à la beauté des dialogues et aux jeux des acteurs, à l'esthétique proposée, à la fluidité des mouvements de caméra et à la lisibilité du montage, au rythme du récit, à la musique et aux bruitages. On jugera ici la qualité et la richesse d'un imaginaire, la puissance de faire monde, de créer des percepts et des affects par le truchement d'un engagement au cœur d'un drame ou d'un mécanisme et c'est à l'art de la prose, soit de la fiction, qu'il faudra remonter (car les dimensions visuelles et musicales sont secondaires, subordonnées à l'art de raconter une histoire ou de créer un univers).

En tant qu'il est un simulacre par lequel le récepteur apprend à se parfaire, il rentre dans l'ensemble des activités qui civilisent et socialisent l'homme moderne. Il naturalise des schèmes technologiques qui lui seront utiles, il contribue au développement de certaines capacités ${ }^{30}$, il participe de l'élaboration de l'habitus et de l'imaginaire contemporains, voire il sensibilise, par la simulation, aux rapports sociaux et aux effets de causalité (les jeux de drague, chers au marché nippon, sont exemplaires, mais les Sims fonctionnent aussi sur ce ressort). Une sorte « d'art de la socialité hypermoderne »-comme l'actualisent de fait les jeux en réseau (par lesquels le joueur peut apprendre à socialiser en ligne, jusqu'avec des inconnus), les jeux de réalité augmentée (type Pokemon Go où les joueurs se rencontreront dans la vraie vie), ou tout jeu narratif dont les personnages se voient de plus en plus animés par des Intelligences Artificielles. La paidia (jeu) se transfigure en une paideia (éducation), et le jeu vidéo serait un vecteur d'adaptation au socius contemporain (le jeu n'est-il pas encore considéré par les éthologues et les psychologues comme un processus mimétique d'apprentissage pour les petits ${ }^{31}$ ?). La source remonterait alors jusqu'à la politesse et le cérémonial, soit l'art de la danse.

En tant qu'il est un loisir mobilisant une certaine dextérité de la part de son récepteur, il divertit tout en l'exerçant, i. e. en perfectionnant l'adresse du joueur dans l'utilisation d'un appareil répondant à ses ordres. Le joueur peaufine ses gestes, son rythme, son attention, sa virtuosité pour dominer les difficultés d'un exercice maintes fois refait et parvenir ainsi à une "trajectoire » singulièrement parfaite, porteuse du style de son 
acteur : il «joue du jeu vidéo ${ }^{32}$ » comme Gould du piano. N'est-ce pas là l'attitude de l'interprète qui renvoie donc à l'art de la musique?

\section{Identification de marqueurs universels de l'art}

\section{L'artiste ou l'auteur}

On reproche parfois au jeu vidéo d'être d'abord une industrie, et que si des grandes entreprises sont réputées, il n'y a pas d'artiste dans leurs équipes (on croirait renouer avec la situation des grands studios de l'âge d'or du cinéma hollywoodien). Or, depuis quelques années, on assiste peut-être à l'émergence de ce qui ressemble à une "politique des auteurs » pour le jeu vidéo : des monographies d'individus paraissent, le nom de Shigeru Miyamoto (créateur de Mario et Zelda) sera bientôt aussi connu que celui de Hergé ou de Lucas, de prestigieuses décorations culturelles sont décernées ${ }^{33}$, les grands pionniers sont en passe de devenir des figures mythiques (Steve Russell, Nolan Bushnell, Alexey Pajitnov), tout un courant de jeux alternatifs enfle en soulignant le nom de leur auteur (Jenova Chen, Jason Rohrer, Jakub Dvorský, Anna Anthropy). Une telle revendication est récente dans l'histoire des jeux vidéo, mais elle concourt à faire émerger cette figure auctoriale du game designer. Or une fois que l'on admet «l'auteur ", les déterminants de l'artiste suivent: son intentionnalité, son style, sa vision, sa compromission dans l'œuvre, son message, etc.

De façon analogue, la politique des studios de développement, bien qu'elle ne concerne sans doute pas les plus gros d'entre eux, assurerait l'équivalent d'une collection ou d'une ligne éditoriale presque aussi connotée artistiquement que celle qui prévaut dans le monde du livre par exemple.

\section{Du mode d'existence de l'œuvre vidéoludique}

En matière d'objectalité, le jeu vidéo ne se distingue nullement de l'art de masse ${ }^{34}$. Comme les CDs ou les DVDs, il fut majoritairement distribué par l'achat de cartouches physiques dont les joueurs devenaient les propriétaires légaux ${ }^{35}$. Avec la tendance actuelle de l'abonnement ou du freemium, qui touche tous les pans de la culture (musique, télévision, etc.), le jeu vidéo à télécharger ou " dans les nuages » va sans doute s'imposer - de sorte qu'on peut parier sur sa dématérialisation physique. Le régime d'existence de l'œuvre «jeu vidéo » n'en restera pas moins celle des arts de masse $^{36}$.

3 La différence et la nouveauté résident dans le fait qu'étant appareillés, ils dépendent de matériels tiers dédiés (consoles, ordinateurs), mais aussi de technologies spécifiques (OS, logiciels, plug-ins) et d'écosystèmes historiques (réseaux sociaux, plateformes, etc. qui subissent des effets de mode importants). Ergo, les jeux vidéo sont périssables ${ }^{37}$. Ce qui s'oppose au régime classique de l'œuvre d'art, éternelle par vocation, contrairement aux produits d'usage, de consommation et de l'action ${ }^{38}$. Et cette caractéristique risque bien d'être fatale à toute possibilité de reconnaissance artistique sur le long terme.

44 Néanmoins, le jeu vidéo a plusieurs moyens de lutter contre cette périssabilité. Premièrement, par l'émulation informatique et son pendant, le retrogaming. Ainsi les jeux vidéo historiques sont-ils toujours accessibles (même si à de nombreux égards déjà 
dénaturés et altérés par l'opération). Le problème de cette solution, c'est qu'elle s'accompagne nécessairement d'un "effet vintage» que les technologies anciennes subissent à cause du progrès de l'œil de l'utilisateur. Les « superbes animations » de tel jeu d'hier nous semblent hachées et baveuses, tout comme la « fluidité » de tel autre ou les "décors somptueux» du troisième. Le jeu émulé a vieilli, irrémédiablement. Difficile alors de retrouver les émotions émerveillées qu'il a pu faire naître dans les yeux des joueurs qui le découvraient au jour de sa sortie. Variante de l'émulation, la réédition du jeu et son portage sur les nouvelles plates-formes pallient ce défaut. À cette occasion, les graphismes sont souvent retravaillés; le canevas originel est conservé, légèrement amendé ou augmenté de chapitres supplémentaires; à moins que le jeu ne soit intégralement à redévelopper. Dernier moyen de résistance temporelle spécifique au jeu vidéo : la sérialité, obtenue par l'exploitation d'une franchise. Ainsi Mario, Zelda et consorts sont toujours au goût du jour parce que les épisodes s'enchaînent sans discontinuer, en suivant les technologies les plus récentes. L'enjeu est de taille pour les développeurs qui doivent chaque fois réaliser l'exploit de conserver la qualité reconnue de l'épisode précédent. Question naïve : dans cent ans, jouera-t-on encore à un Zelda ? Et dira-t-on alors qu'on « joue à Zelda»?

Figure 1.a. Copie d'écran du jeu Another World, affichage originel

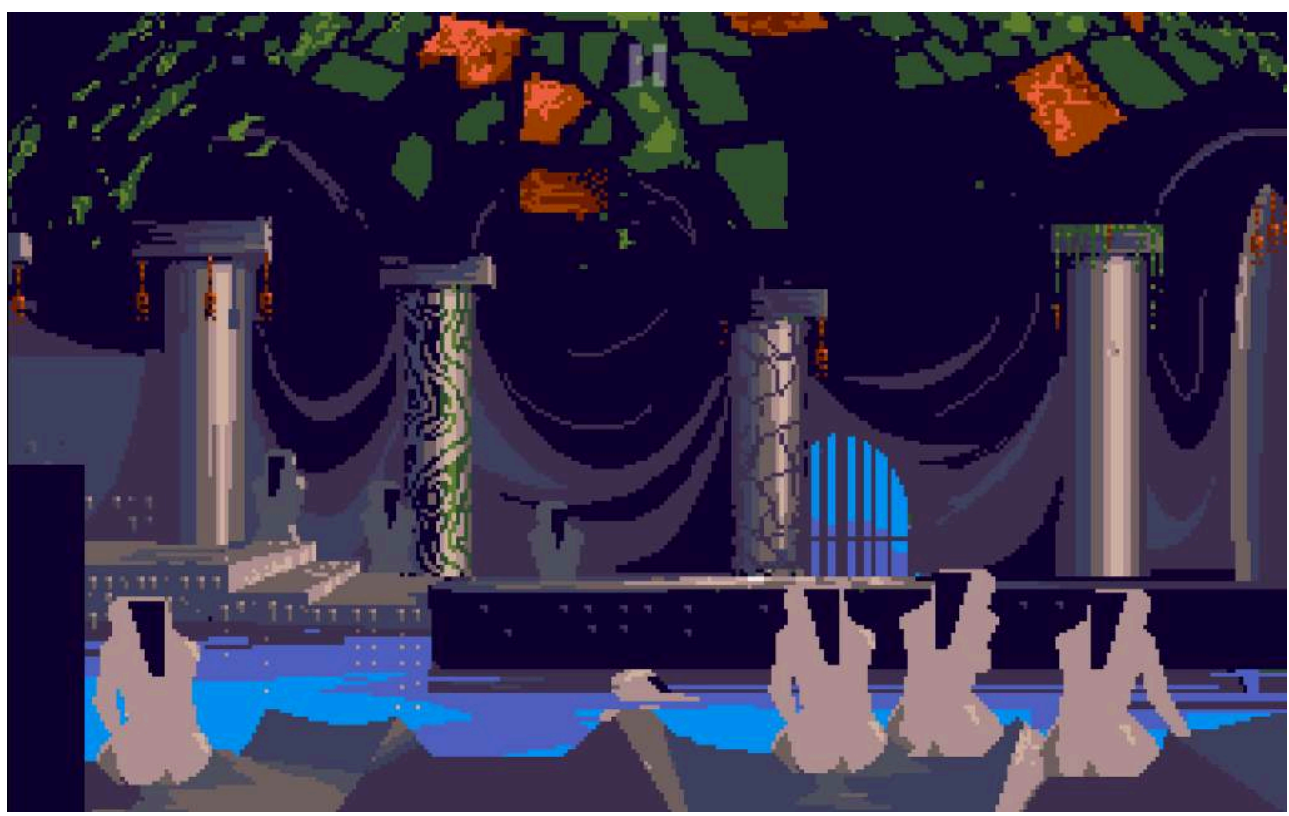

Source : Éric Chahi, Another World, Paris, Delphine Software, 1991 (crédits image : Pascal Krajewski) 
Figure 1.b. Copie d'écran du jeu Another World, affichage remasterisé

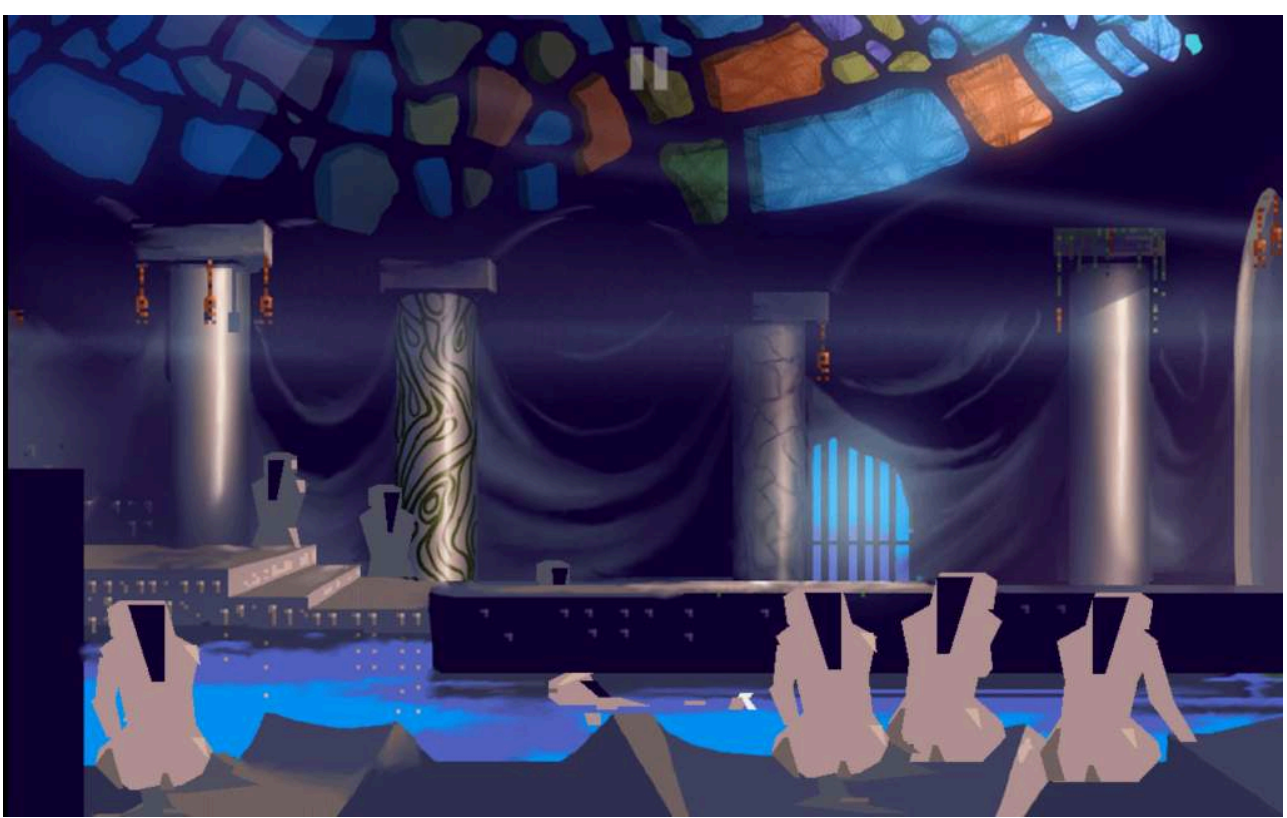

Disponible sous forme d'application sur Android A

Source : Éric Chahi, Paris, Dotemu, 2011 (crédits image : Pascal Krajewski)

\section{La diversification des plaisirs esthétiques} attitude esthétique - voilà qui clôt le débat ! Cela dit... Pour rêver à la possibilité d'un terrain d'entente entre ces deux-là, il faudrait donc imaginer soit la coexistence harmonieuse de ces deux attitudes dans l'activation d'un même objet, soit l'existence d'une composante ludique au sein de l'attitude esthétique (assez mal définie somme toute), soit la production d'effets esthétiques à l'intérieur de l'attitude ludique (qu'il faudrait nourrir).

Cette dernière solution est la plus suivie et va consister à élargir la gamme des affects pouvant être suscités par le jeu vidéo. Le jeu vidéo sera de l'art s'il est capable de produire des émotions, qui soient autre chose que de la peur, de la tension et du relâchement nerveux. Ou, pour le dire dans un langage de neurologues : au-delà des " émotions ", d'accéder aux « sentiments ».

La quête du sentiment devient en effet une piste privilégiée pour tirer le jeu vidéo vers sa maturité et vers l'art. Ici, le jeu Ico (Sony, 2001) pourrait faire figure de modèle. Dans ce jeu, le joueur incarne un garçon devant accompagner une princesse vers la sortie du château qui la retient enfermée. L'avatar du joueur se déplace physiquement avec sa compagne et doit parfois l'abandonner pour progresser au risque qu'elle ne se fasse rattraper par les forces supposées obscures du lieu. Heavy rain (Quantic dream, 2010) insiste sur les séquences initiales dévolues aux interactions entre un père de famille (incarné par le joueur) et ses enfants. La scène de disparition de l'enfant dans le grand magasin est régulièrement invoquée comme modèle de moments forts où le joueur éprouve une angoisse intime qui n'est pas celle des jeux d'horreur. Concluons avec la mort d'Aerith dans Final fantasy VII dont on sait qu'elle arrache d'authentiques larmes aux joueurs qui la vivent. 

allégations de principe qui ne se révèlent in fine qu'idéologiques, regardons ce qui existe et admirons les jeux vidéo pour ce qu'ils sont avant de spéculer sur ce qu'ils disent sur le devenir du jeu vidéo. Attitude pragmatique et honnête qui devrait permettre de se mettre au moins d'accord sur ce dont on parle, et sur ce qu'il faut considérer pour juger en conscience. Malheureusement, le risque est grand d'aboutir à plusieurs listes assez incompatibles. meilleures ventes du secteur : Zelda, Mario, World of Warcraft, GTA, Final Fantasy, Street Fighter, etc. Ces noms sont, partiellement au moins, connus des plus ignares d'entre nous - même si ce ne sont pas toujours à proprement parler des noms d'œuvres singulières, mais de licences qu'il faudrait chaque fois préciser. Gamasutra $^{39}$ ou un journal comme Les Inrocks sont capables dans leurs palmarès annuels de saluer le blockbuster réussi (The Legend of Zelda: Breath of the Wild en 2017) comme d'élire le jeu indépendant (Gorogoa en 2017). De même, des classements de développeurs mettent régulièrement en avant de petits studios, dont les jeux souvent passés inaperçus du grand public, n'en sont pas moins des viviers de tendances et de schèmes ludiques (Laundry bear games en 2017, Tale of tales en 2015). Le Triennal game festival de Milan supportera-t-il un jour la comparaison avec le festival de Cannes?

Suivra la liste des historiens, à la recherche du jeu qui créa le genre. Dans ce cas, Wolfenstein 3D (1992) sera cité, plutôt que son successeur Doom (pourtant à la source de l'appellation des jeux «doom-like»), père d'une foisonnante progéniture de jeux aujourd'hui appelés FPS (First Person Shooter), que les plus jeunes joueurs ne connaissent qu'à travers Call of Duty ou Halo ${ }^{40}$. De même Alone in the dark (1992) a créé le genre horreur qu'on rattache aujourd'hui plus volontiers à la magistrale saga des Resident Evil. Tetris, Pac-man, Spacewar ou Pong marquent les étapes obligées de la grande histoire des 
jeux vidéo même si leur notoriété et leur diffusion font pales figures comparé à ce que leurs ancêtres connurent.

La liste des esthéticiens sera encore différente, désireuse de débusquer des jeux artistiques, d'avant-garde et un peu alternatifs, qui explorent ce que le jeu vidéo traditionnel n'explore précisément pas. Que l'on parle de « jeux artistiques » (art games) ou au contraire "d'art vidéoludique " (game art) - et aux nuances impliquées près -, seront retenus soit des jeux vidéo ayant réussi à sortir sur de grandes plates-formes bien qu'explorant de nouvelles fibres esthético-ludiques (Ico, Myst, Black rain, Flower, Little big planet, Braid, Rez), soit des " petits » jeux issus de et cantonnés à la scène indépendante (souvent en flash), mal diffusés, et même assez éloignés des plaisirs vidéoludiques classiques (Passage, Machinarium, PaRappa the Rapper). La capacité "critique " de la discipline (au-delà des simples jeux de citations, d'influences, de décalages ou d'intertextualité souvent utilisés), visible dans la conception d'œuvres interrogeant le médium de l'intérieur, pourrait servir de preuve décisive. C'est chose faite et très bien faite avec Evoland (entre autres), créé en 2012 puis porté sur plateformes nomades : le joueur y incarne un héros d'un jeu d'aventure qui, en guise de trésor, débloque des ressources graphiques et informatiques du jeu, passant d'un vieux jeu des années 1980 à un jeu contemporain, en activant les couleurs, le scrolling, la 3D, etc.

Le spectre est ainsi couvert, allant des jeux les plus grands publics, les blockbusters, jusqu'aux jeux les plus esthètes ou intellectuels qui n'intéressent qu'un public de franges. Cela pourrait assez ressembler à ce qui se passe dans n'importe quel art de masse, où les critiques et les box-offices tombent rarement d'accord sur des titres communs. On pourrait ainsi retrouver la situation du cinéma, dont l'histoire retient à la fois les films à grand succès (Star Wars, Titanic, La Grande Vadrouille), les films accessibles de grands réalisateurs (Hitchcock, Mankiewicz, Lang, Kurosawa, Carné) et les films d'auteur « pour cinéphiles avertis » (Dreyer, Tarkovski, Herzog, Rivette, Cassavetes).

\section{Mise en avant de principes compatibles avec l'art}

Finalement, la discussion pourra se placer sur le plan des «principes ». Dans l'absolu cela nécessiterait de recourir à une définition de l'art. Mais qui encore oserait en proposer une, à l'heure où sa dé-définition orchestrée par les arts modernes et contemporains $\mathrm{s}^{41}$ a sacré le règne $\mathrm{du}$ "tout peut faire art». Sitôt une définition proposée, il sera aisé à l'œil sagace de dénicher moult œuvres exemplaires, trônant aux meilleures places de nos musées d'art, pour faire mentir l'audacieux.

Une version faible pourra consister à mettre en avant un certain nombre de principes que devrait suivre l'objet se rêvant œuvre d'art : la transcendance, la poésie, le fairemonde, le sublime, l'émotion, le sacré, l'Idée, le Beau, le mystère, etc. Cette démarche est sans doute plus continentale qu'anglo-saxonne, elle n'en est pas moins parfaitement légitime. Bien sûr, il n'y a aucune façon de s'accorder sur ces principes, il n'y a que des manières de les défendre avec plus ou moins de conviction. Relevons quelques attitudes qui émaillèrent les polémiques à cet égard et remarquons qu'elles dessinent trois genres de débats, selon l'accord implicite qui se fait sur leurs termes.

L'art (n') est (pas) de l'art, parce qu'il est (in)capable "d'émouvoir» le joueur. Il ne s'agit pas de renvoyer aux émotions strictement ludiques - stress, frisson, agacement, joie - mais à la gamme des sentiments, des affects : intimité, famille, enthousiasme, 
chagrin, empathie ${ }^{42}$. C'est la piste la plus suivie aujourd'hui par les défenseurs des jeux vidéo comme art. Il s'agit alors pour les uns d'insister sur des exemples prouvant que l'expression affective n'est pas inaccessible aux jeux vidéo, face à des détracteurs qui le nieront. Il n'y a là aucune mésentente sur les termes : le joueur qui se dit profondément ému par telle scène ou tel jeu sera considéré comme un peu niais par son débatteur qui goûte des plaisirs plus subtils mais qui souvent, avouons-le, ne goûte pas celui du jeu vidéo (de fait, Céline Dion me tire des larmes, [parce que] cela reste de la guimauve). Un terrain commun pourrait les réunir si on déplaçait l'accent sur le potentiel émotionnel des jeux vidéo.

L'art (n) est (pas) de l'art, parce qu'il est « ouvert ${ }^{43}$ ». Intéressant de voir qu'un même argument peut-être lu de façon diamétralement opposée. Certains ont reproché au jeu vidéo son côté interactif, permettant d'aboutir à des versions différentes de l'histoire et qui donc ne saurait exprimer la vision, l'intentionnalité de l'auteur. Pas une seule ligne de Roméo et Juliette ne saurait être touchée sans que l'œuvre ne s'en ressente - et l'art interactif est une contradictio in adjecto ${ }^{44}$. Au contraire, pour d'autres, cet argument plaidera dans le sens d'une richesse du monde proposé par le programme et d'une immersion offerte au joueur baignant au cœur de l'œuvre, la vivant de l'intérieur, l'éprouvant jusque dans sa chair. Débat insoluble puisque s'y renvoient dos à dos deux conceptions d'un même terme.

61 L'art (n) est (pas) de l'art, parce qu'il est (in)capable de «transcendance ». Ce type d'argumentation est piquant puisqu'il invite dans le débat un nouveau vocable indéfinissable (à côté de celui d'art et même de jeu vidéo ${ }^{45}$ ). Admettons que ce troisième terme renvoie à un moyen d'élever l'homme au-dessus de son humaine condition ou de la réfléchir en articulation avec tout ce qui le dépasse, l'au-delà et l'éternité. Là encore, les défenseurs et les détracteurs ont peu de chance de se mettre d'accord, l'un considérant la valeur atteinte dans ce que l'autre ne tolérera sans doute que comme une caricature. Le jeu Passage de Jason Rohrer (2007) offrirait ici un recours ambigu. Petit jeu d'à peine cinq minutes, il est censé figurer le trajet d'une vie jusqu'à son terme. C'est très certainement un intéressant programme informatique, offrant une métaphore de l'existence, mais pour autant, il achoppe à tous les égards : d'un côté, la maturité de son thème ne suffit pas à en faire une œuvre d'art (il s'en faut de beaucoup) ; de l'autre, sa nature informatique ne saurait masquer qu'il est à peine un jeu vidéo, que les joueurs ne sauraient y prendre le moindre plaisir et in fine de conclure que, s'il en est un, c'est un très mauvais jeu vidéo. Par contre, en tant que tentative pour ouvrir le domaine (et le débat), c'est une expérience très passionnante (comme Fountain de Duchamp est à peine de l'art ou est une très mauvaise œuvre d'art - mais n'en reste pas moins l'œuvre la plus importante $d u x^{e}$ siècle!). Ces jeux alternatifs seraient peut-être aux jeux vidéo ce que le Nouveau roman fut au roman...

\section{Conclusion : « Faire comme si »}

Derrière chacune de ces approches, se cachent des attitudes diverses vis-à-vis de l'art: définition institutionnelle de l'art, attitude opportuniste, approche relativiste, démarche positive, revendication idéologique (qui statue sur ce qu'est l'art et donc sur sa valeur). En outre, persévérer dans ce questionnement est l'assurance de renouer avec les polémiques stériles qui touchèrent la photographie, puis le cinéma, puis la bande dessinée. Classiquement, le débat s'organisera toujours de la même façon: les 
détracteurs diront que ce n'est pas de l'art, parce que cela ne peut susciter les valeurs archaïques, cultuelles, transcendantes de l'art; les défenseurs diront qu'au contraire, elles y arrivent sous une forme rafraîchie.

Walter Benjamin, pour le cinéma, les renvoie dos à dos, et s'attache plutôt à montrer ce qui a changé dans le régime de perception de l'homme avec l'arrivée de la nouvelle technique : la réception distraite, l'effet de choc d'un art plus tactile que visuel ${ }^{46}$. Le nouvel entrant s'est imposé comme art pour des raisons de fait - et l'art a dû se reconfigurer, s'augmenter de nouvelles modulations, afin de pouvoir l'accueillir, de droit.

Ici aussi, la solution pourrait survenir en adoptant une attitude naïve, en «faisant comme si ». On pourrait réfléchir à l'objet jeu vidéo, en « faisant comme si » c'était de l'art, c'est-à-dire en adoptant le biais et la méthode de l'esthéticien - sans trancher en amont sur sa valeur ou sa fonction. Dès lors, nous pourrions mener l'enquête en trois temps : en réfléchissant au régime créatif spécifique du jeu vidéo, en analysant l'œuvre vidéoludique et en proposant une esthétique du jeu vidéo. Au sortir de ces analyses, se dessinerait une image qui ne serait pas un «portrait du jeu vidéo en art " mais un «portrait du jeu vidéo par l'esthétique » - et c'est cette figure que l'on pourrait ensuite proposer au jugement pour délibérer sur la possibilité qu'il fasse ou non partie de la grande famille des arts.

\section{BIBLIOGRAPHIE}

Aarseth Espen, Cybertext: Perspectives on ergodic literature, Baltimore-London, Johns Hopkins University Press, 1997.

Aarseth, Espen, Game Studies: The International Journal of Computer Game Research, en ligne: http://gamestudies.org

Alain, Système des Beaux-arts [1926], Paris, Gallimard, 1953.

Albinet, Marc, Concevoir un jeu vidéo, Fyp, 2015.

Antonelli, Paola, « Video Games: 14 in the Collection, for Starters », dans Inside/Out, A MOMA Blog, 29/11/2012, en ligne : https://www.moma.org/explore/inside_out/2012/11/29/video-games-14in-the-collection-for-starters/

Arendt Hannah, La Crise de la culture, Paris, Gallimard, 1972.

Arsenault Dominic, « Video Game Genre, Evolution and Innovation », dans Eludamos. Journal for Computer Game Culture, 2009.

Benjamin, Walter, L'Euvre d'art à l'époque de sa reproductibilité technique, Paris, éditions Allia, 2011.

Blanchet, Alexis, Les jeux vidéo au cinéma, Paris, éditions Armand Colin, 2012.

Bonhomme, Stello et Talon-Hugon, Carole (sld), Nouvelle revue d'esthétique. $N^{\circ} 11$ : Esthétique des jeux vidéo, Paris, Puf, 2013, en ligne : https://www.cairn.info/revue-nouvelle-revue-desthetique-2013-1.htm 
Coville Marion, La Construction du jeu vidéo comme objet muséal : le détournement d'un objet culturel et technique de son cadre d'usage initial et son adaptation au contexte muséal : étude de cas dans un centre de sciences [Thèse], Paris, Université Paris I, 19/10/2016.

Crawford Chris, The Art of computer game design, McGraw-Hill/Osborne Media, 1984 [en ligne].

Dehaene Stanislas, Apprendre! Les talents du cerveau, le défi des machines, Paris, éditions Odile Jacob, 2018.

Deleuze Gilles, Qu'est-ce que la philosophie ?, Paris, Éditions de Minuit, 1991

Didi-Huberman Georges, Devant l'image, Paris, Éditions de Minuit, 1990.

Ebert, Roger, « Games VS Art: Ebert VS Barker », dans Roger Ebert Journal, 21/07/2007

Frasca Gonzalo, « Simulation versus Narrative: Introduction to Ludology », dans Wolf Mark J.P. et Bernard Perron (dir.), Video/Game/Theory, Routledge, 2003.

Hegel, Georg Wilhelm Friedrich, Esthétique, Tome 1, Paris, Librairie Générale Française, 1997.

Hurel, Pierre-Yves, « Le passage du jeu à la création : le cas du jeu vidéo amateur », dans Sciences $d u \mathrm{Jeu}, \mathrm{n}^{\circ}$ 7, Les marges du jeu, 2017, en ligne : https://journals.openedition.org/sdj/766

Jones, Jonathan, « Sorry MoMA, video games are not art », dans The Guardian, 30/11/2012

Juul Jesper, Half Real: Video Games between Real Rules and Fictional Worlds, Cambridge, MIT Press, 2005.

Kant Emmanuel, Critique de la faculté de juger, Paris, éditions Aubier, 1995.

Koster Ralph, Theory of fun for game design, O’Reilly, 2013.

Krajewski Pascal (sld), Convocarte. No. 6-7: Ars Ludens : L'art, le jeu, le ludique, Lisbonne, FBAUL, 2019, en ligne : http://convocarte.belasartes.ulisboa.pt/index.php/category/httpconvocartebelasartes-ulisboa-ptrevistaconvocarte/

Krichane, Selim (sld), Décadrage, n 39, Jeu vidéo et cinéma, Lausanne, automne, 2018.

Le Diberder, Alain et Frédéric, Qui a peur des jeux vidéos ?, Paris, éditions La découverte, 1993.

Loos, Adolf, Ornement et crime, Paris, Payot\&Rivages, 2003.

Maeda, John, « Videogames do belong in the Museum of Modern art », dans Wired, 04/12/2012.

Manovich, Lev, Le langage des nouveaux médias, Dijon, Presses du réel, 2010.

Mckenzie, Mark, Théorie du gamer, Paris, éd. Amsterdam, 2019.

Melancon, Benoît et Perron, Bernard (sld), Sciences du jeu, nº 6, L'art en jeu ou le jeu de l'art, 2016, en ligne : https://journals.openedition.org/sdj/666

Michaud, Yves, L'Art à l'état gazeux : essai sur le triomphe de l'esthétique, Paris, éditions Pluriel, 2011.

Millar Susanna, La Psychologie du jeu chez les enfants et les animaux [1968], Paris, éditions Payot, 2002.

Montembeault Hugo et Maxime Deslongchamp-Gagnon, Profil ludique, sur Soundcloud, en ligne : https://soundcloud.com/profil-ludique

Moriarty, Brian, « A designer's take: MoMA is wrong to pretend video games are art », dans The Guardian, 07/03/2013.

Murray Janet, Hamlet on the Holodeck: The Future of Narrative in Cyberspace, Free Press, 1997. 
Perron Bernard, « Jeu vidéo et émotions », dans Genvo Sébastien (sld), Le game design de jeux vidéo. Approches de l'expression vidéoludique, Paris, éditions L'Harmattan, 2006.

Poole, Steven, Trigger happy: Videogames and the Entertainment Revolution, Arcade Publishing, 2000 [en ligne].

Pouivet Roger, L'Euvre d'art à l'âge de sa mondialisation, Bruxelles, éditions La Lettre volée, 2003.

Rosenberg Harold, La Dé-définition de l'art, Nîmes, éditions Jacqueline Chambon, 1998.

Ruffat Samuel et Hovig Ter Minassian, Les Jeux vidéo comme objet de recherche, Paris, éditions Questions Théoriques, 2012.

Schopenhauer Arthur, Le Monde comme volonté et comme représentation, Paris, Puf, 2004.

Ubm, Gamasutra - The Art \& Business of Making Games, en ligne: http://www.gamasutra.com/

Tavinor, Grant, « Definition of videogames », Contemporary Aesthetics, vol. 6, 10/01/2008, en ligne : http://www.contempaesthetics.org/newvolume/pages/article.php?articleID=492

Tavinor Grant, « Video games as mass art », dans Contemporary Aesthetics, vol. 9, 2011, en ligne : https://contempaesthetics.org/newvolume/pages/article.php ?articleID =616

Triclot Mathieu, Philosophie des jeux vidéo, Paris, éditions La découverte, 2011.

Vial Stéphane, Court traité du design, Paris, Puf, 2010.

\section{NOTES}

1. Quelques pistes esthétiques sont suivies dans Pascal Krajewski (sld), Convocarte, $\mathrm{n}^{\circ}$ 6-7, Ars Ludens: L'art, le jeu, le ludique, Lisbonne, FBAUL, 2019, en ligne: http:// convocarte.belasartes.ulisboa.pt/index.php/category/httpconvocarte-belasartes-ulisboaptrevistaconvocarte/.

2. Par exemple avec Stello Bonhomme et Carole Talon-Hugon (sld), Nouvelle revue d'esthétique, $\mathrm{n}^{\circ}$ 11, Esthétique des jeux vidéo, Paris, Puf, 2013, en ligne : https://www.cairn.info/revue-nouvellerevue-d-esthetique-2013-1.htm.

3. Gilles Deleuze, Qu'est-ce que la philosophie ?, Paris, Éditions de Minuit, 1991.

4. Georg Wilhelm Friedrich Hegel, « $1^{\text {re }}$ partie : de l'idée de beau artistique ou de l'idéal », dans Esthétique, Tome 1, Paris, Librairie Générale Française, 1997, p. 177.

5. Arthur Schopenhauer, «Livre III. $\$ 52$ », dans Le Monde comme volonté et comme représentation, Paris, Puf, 2004, p. 341.

6. Voir par exemple, Georges Didi-Huberman, « 2. L'art comme renaissance et l'immortalité de l'homme idéal ", dans Devant l'image, Paris, Éditions de Minuit, 1990, p. 65-103 et surtout p. 94 sqq. 7. «Le mot disegno était un mot de l'esprit autant qu'un mot de la main", résume DidiHubermann, rappelant les termes de Vasari : «Procédant de l'intellect, le dessin, père de nos trois arts - architecture, sculpture et peinture - extrait à partir de choses multiples un jugement universel. Celui-ci est comme une forme ou idée de toutes les choses de la nature, toujours très singulière dans ses mesures. [...] Et de cette connaissance naît un certain concept ou jugement qui forme dans l'esprit cette chose qui, exprimée par la suite avec les mains, se nomme le dessin. On peut en conclure que ce dessin n'est rien d'autre que l'expression apparente et la déclaration du concept que l'on possède dans l'esprit, ou de ce que d'autres ont imaginé dans leur esprit et fabriqué dans l'idée ", Georges Didi-Huberman, Devant l'image, op. cit., p. 96.

8. Adolf Loos, Ornement et crime, Paris, éditions Payot \& Rivages, 2003. 
9. Yves Michaud, L'Art à l'état gazeux: essai sur le triomphe de l'esthétique, Paris, éditions Pluriel, 2011.

10. Lev Manovich, Le Langage des nouveaux médias, Dijon, Presses du réel, 2010.

11. Stéphane Vial, Court traité du design, Paris, Puf, 2010, p. 75-98.

12. Nous traduisons Paola Antonelli, "Video Games: 14 in the Collection, for Starters ", dans Inside/Out, A MOMA Blog, 29/11/2012, en ligne: https://www.moma.org/explore/inside_out/ 2012/11/29/video-games-14-in-the-collection-for-starters/.

13. Smithonian American Art Museum, "The Art of Video Games ", en ligne: https:// americanart.si.edu/exhibitions/games.

14. John Maeda, «Videogames do belong in the Museum of Modern art », dans Wired, 04/12/2012.

15. Jonathan Jones, "Sorry MoMA, video games are not art ", dans The Guardian, 30/11/2012. Brian Moriarty, «A designer's take: MoMA is wrong to pretend video games are art ", dans The Guardian, 7/03/2013.

16. Ce genre de questionnements a déjà été soulevé pour les jeux vidéo de type «simulateurs de balade». Profil ludique, «S01E03 - 'Le walking simulator: Jeu ou non-jeu ?' : Telle est la question ", Soundcloud, 01/11/2017, en ligne: https://soundcloud.com/profil-ludique/profilludique-s01e03-jeu-ou-non-jeu-telle-est-la-question.

17. Au-delà des musées spécifiquement dédiés au jeu ou au jeu vidéo.

18. Sans parler des problèmes strictement muséographiques que pose l'exposition d'un objet informatique, interactif, en réseau, jouable, etc. Voir Marion Coville, La Construction du jeu vidéo comme objet muséal : le détournement d'un objet culturel et technique de son cadre d'usage initial et son adaptation au contexte muséal: étude de cas dans un centre de sciences [thèse], université Paris I, 19/10/2016.

19. Voir par exemple : Janet Murray, Hamlet on the Holodeck: The Future of Narrative in Cyberspace, Free Press, 1997.

20. Voir par exemple : Jesper Juul, Half Real: Video Games between Real Rules and Fictional Worlds, Cambridge, MIT Press, 2005.

21. Sur cette distinction classique, voir encore : Gonzalo Frasca, «Simulation versus Narrative: Introduction to Ludology ", dans Mark J.P. Wolf et Bernard Perron (sld), Video/Game/Theory, Routledge, 2003.

22. Et même, plus inattendu, En attendant Godot de Beckett.

23. Pour aller plus loin : Alexis Blanchet, Les Jeux vidéo au cinéma, Paris, éditions Armand Colin, 2012 ; Selim Krichane (sld), Décadrages, $\mathrm{n}^{\circ}$ 39, Jeu vidéo et cinéma, Lausanne, Automne, 2018.

24. Espen Aarseth, Cybertext: Perspectives on ergodic literature, Baltimore-London, Johns Hopkins University Press, 1997.

25. Ralph Koster, Theory of fun for game design, O’Reilly Media, 2013.

26. Chris Crawford, The Art of computer game design, McGraw-Hill/Osborne Media, 1984; Marc Albinet, Concevoir un jeu vidéo, FYP Éditions, 2015.

27. Emmanuel Kant, «§51: De la division des beaux-arts », dans Critique de la faculté de juger, Paris, Aubier, 1995, p. 310-311.

28. Alain, Système des Beaux-arts [1926], Paris, Gallimard, 1953.

29. À voir aussi : Martin Picard, « Les enjeux esthétiques du jeu vidéo : entre art, stylistique et interactivité ", dans Sciences du jeu. $N^{\circ} 6$ : L'art en jeu ou le jeu de l'art, 2016, en ligne : https:// journals.openedition.org/sdj/712\#tocto1n2.

30. Les neurologues assurent que l'attention des enfants gagne à user des jeux vidéo. Stanislas Dehaene, Apprendre! Les talents du cerveau, le défi des machines, Paris, Odile Jacob, 2018, p. 228-229.

31. Susanna Millar, La Psychologie du jeu chez les enfants et les animaux [1968], Paris, Payot, 2002.

32. L'expression est peut-être littérale dans le cas des jeux autorisant l'implication créative de leurs joueurs. Pierre-Yves Hurel, « Le passage du jeu à la création : le cas du jeu vidéo amateur », 
dans Sciences $d u \mathrm{Jeu}, \mathrm{n}^{\circ} 7$, Les marges $d u \mathrm{jeu}, 2017$, en ligne : https://journals.openedition.org/sdj/ 766.

33. En 2006, Shigeru Miyamoto, Michel Nacel et Frédérick Raynal reçoivent l'insigne de Chevalier de l'ordre des Arts et des Lettres (France).

34. Grant Tavinor, "Video games as mass art ", dans Contemporary Aesthetics, vol. 9, 2011, en ligne : https://contempaesthetics.org/newvolume/pages/article.php ?articleID =616.

35. Pour une histoire complète du jeu vidéo, voir : Mathieu Triclot, Philosophie des jeux vidéo, Paris, La découverte, 2011.

36. Roger Pouivet, L'Euvre d'art à l'âge de sa mondialisation, Bruxelles, La Lettre volée, 2003.

37. Ils sont pour cette triple raison (matérielle, logicielle et contextuelle) bien plus périssables que les autres arts médiatiques. Peut-on encore jouer et apprécier Second Life (s'il fut jamais un jeu)?

38. Hannah Arendt, La Crise de la culture, Paris, Gallimard, 1972, p. 266-268.

39. Voir ses « Top 10 games of the year » et ses « Tome 10 game developers of the year».

40. Pour aller plus loin : Dominic Arsenault, "Video Game Genre, Evolution and Innovation », dans Eludamos. Journal for Computer Game Culture, 2009.

41. Harold Rosenberg, La dé-définition de l'art, Nîmes, ed. Jacqueline Chambon, 1998.

42. Bernard Perron, "Jeu vidéo et émotions ", dans Sébastien Genvo (sld), Le game design de jeux vidéo. Approches de l'expression vidéoludique, Paris, L'Harmattan, 2006.

43. Roger Ebert, "Games VS Art: Ebert VS Barker », dans Roger Ebert Journal, 21/07/2007, en ligne.

44. Cet argument reste assez faible pour le joueur qui sait très bien que l'ouverture des jeux n'est pas si large que cela. Si Roméo et Juliette était un jeu vidéo, la bataille Montaigu-Capulet ne pourrait pas plus être évitée que la mort tragique des héros.

45. Grant Tavinor, « Definition of videogames », dans Contemporary Aesthetics, Vol 6, 10/01/2008, en ligne : http://www.contempaesthetics.org/newvolume/pages/article.php?articleID=492.

46. Walter Benjamin, L'Euvre d'art à l'époque de sa reproductibilité technique, Paris, éditions Allia, 2011.

\section{RÉSUMÉS}

Se demander si le jeu vidéo est un art reviendrait sans doute à se demander s'il a accouché d'œuvres d'art ou s'il est susceptible de créer des sentiments esthétiques. Faisant le tour des différents discours de légitimation qui ont eu cours, nous pouvons observer un large panel de plaidoyers : l'enrôlement dans un contexte artistique institutionnel, l'analogie avec des arts connexes reconnus, l'intégration dans un système des beaux-arts élargi, l'identification de marqueurs universels de l'art, l'élection de (proto) chefs d'œuvres ou la mise en avant de principes compatibles avec l'art.

Cependant, aucune de ces méthodes ne pourra convaincre le détracteur, et Walter Benjamin pourrait fournir une attitude plus sage et pragmatique : faire comme si le jeu vidéo était un art pour voir ce qu'il fait à notre sensibilité. 
INDEX

Mots-clés : jeu vidéo, esthétique, histoire, théorie, game studies

\section{AUTEUR}

\section{PASCAL KRAJEWSKI}

Docteur en sciences de l'art, Pascal Krajewski est chercheur associé à la Faculté des Beaux-Arts de l'université de Lisbonne (Laboratoire CIEBA). Son travail de recherche porte sur l'art des nouveaux médias, la technologie, les appareils, les images, la bande dessinée. Il est l'auteur de plusieurs ouvrages dont L'Art au risque de la technologie (2013), L'Ordre technologique (2016) et Art, médium, média (2018). 